07

\title{
Локализация электронов верхних долин в узкозонном канале - возможный дополнительный механизм увеличения тока в DA-DpHEMT
}

\author{
(C) А.Б. Пашковский, С.А. Богданов
}

АО НПП „Исток“ им. Шокина, Фрязино, Московская обл., Россия

E-mail: solidstate10@mail.ru

Поступило в Редакцию 13 июня 2019г.

В окончательной редакции 26 июня 2019 г.

Принято к публикации 26 июня 2019 г.

\begin{abstract}
Проведена теоретическая оценка влияния локализации электронов верхних долин в узкозонном канале транзисторных гетероструктур $\mathrm{Al}_{x} \mathrm{Ga}_{1-x} \mathrm{As}-\mathrm{GaAs}$ с двусторонним легированием на величину всплеска дрейфовой скорости. Показано, что для транзисторных гетероструктур с донорно-акцепторным легированием, в которых доля электронов, перешедших из узкозонного канала в широкозонный материал, меньше, чем в обычных структурах, за счет локализации электронов верхних долин в узкозонном канале в ряде случаев увеличение дрейфовой скорости может достигать $15 \%$. Исследованный эффект может являться дополнительным механизмом увеличения тока в транзисторах на основе гетероструктур с донорноакцепторным легированием.
\end{abstract}

Ключевые слова: донорно-акцепторное легирование, верхние долины, узкозонный канал, всплеск дрейфовой скорости.

DOI: 10.21883/PJTF.2019.20.48385.17925

При нелокальном транспорте горячих электронов в транзисторных гетероструктурах $\mathrm{Al}_{x} \mathrm{Ga}_{1-x} \mathrm{As}-\mathrm{GaAs}$ с селективным легированием их динамика может иметь достаточно сложный характер [1-6]. Из-за специфических особенностей процессов переноса носителей заряда могут проявляться механизмы, несвойственные объемным материалам и нелегированным гетероструктурам [7]. Рассмотрим один из них на примере транзисторных гетероструктур с двусторонним легированием. При разогреве электронов в квантовых ямах транзисторных гетероструктур начинаются их переходы в верхние долины, где эффективная масса электронов гораздо больше, а температура сильно падает. Кроме того, для электронов в $L$-долине существенно изменится рельеф дна зоны проводимости: потенциальная яма в узкозонном материале канала станет более мелкой. При этом в не слишком сильных полях тепловая энергия тяжелых электронов в $L$-долине будет меньше разрыва зон по $L$-долине. Это приведет к тому, что бо́льшая часть этих электронов до того, как их температура сильно увеличится, должна локализоваться в узкозонном материале, где рассеяние существенно ниже, чем в широкозонном. Из-за особенностей потенциального рельефа в области канала локализация электронов особенно сильно должна проявляться в структурах с донорно-акцепторным легированием (DA-DpHEMT) [8], где барьеры на основе $p-n$-переходов препятствуют выходу электронов из узкозонного канала. Далее представлена оценка влияния этого эффекта на динамику разогрева электронов.
Для расчетов используем простую феноменологическую модель [7]. При анализе нелокального разогрева электронов предполагается, что в перпендикулярном поверхности направлении устанавливается состояние термодинамического равновесия, определяемое температурой электронов. Потенциальный рельеф и уровни размерного квантования в этом направлении определяются на основе численного самосогласованного решения уравнений Шредингера и Пуассона. Электроны в канале задаются общим ансамблем, для которого законы сохранения энергии и импульса описываются с помощью уравнений, аналогичных системе, приведенной в [9]. Для простоты заменим обрамляющие узкозонный канал широкозонные слои из одинакового материала одним эффективным слоем с соответствующей суммарной вероятностью нахождения электронов в нем. Для двух слоев система уравнений законов сохранения импульса и энергии с учетом вида времен релаксации в предположении, что частота рассеяния электронов задается в виде $v=v_{1} p_{1}+v_{2} p_{2}$ (где $p_{1}$, $p_{2}$ и $v_{1}, v_{2}$ - вероятности нахождения электронов и их частоты рассеяния в узкозонном слое канала и в более широкозонных слоях, обрамляющих канал), имеет вид [7]:

$$
\begin{gathered}
m^{*}(\varepsilon)=m_{1}^{*}(\varepsilon) p_{1}+m_{2}^{*}(\varepsilon) p_{2}, \\
\frac{\partial\left(m^{*}(\varepsilon) V\right)}{\partial t}=q \\
\times\left(E-\frac{m^{*}(\varepsilon) V\left(m_{1}^{*}(\varepsilon) V_{s 1}(\varepsilon) E_{s 2}(\varepsilon) p_{2}+m_{2}^{*}(\varepsilon) V_{s 2}(\varepsilon) E_{s 1}(\varepsilon) p_{1}\right)}{m_{1}^{*}(\varepsilon) V_{s 1}(\varepsilon) m_{2}^{*}(\varepsilon) V_{s 2}(\varepsilon)}\right),
\end{gathered}
$$




$$
\begin{aligned}
& V_{s}(E)= \\
& =\frac{m_{1}^{*}(E) V_{s l}(E) m_{2}^{*}(E) V_{s 2}(E)}{\left(m_{1}^{*}(E) p_{1}+m_{2}^{*}(E) p_{2}\right)\left(m_{1}^{*}(E) V_{s 1}(E) p_{2}+m_{2}^{*}(E) V_{s 2}(E) p_{1}\right)}, \\
& \quad \frac{\partial \varepsilon}{\partial t}=q\left(E V-V_{s}(\varepsilon)\left(E_{s 1}(\varepsilon) p_{1}+E_{s 2}(\varepsilon) p_{2}\right)\right)
\end{aligned}
$$

Здесь $q, V, m^{*}, \varepsilon-$ заряд, скорость, усредненная эффективная масса и энергия электронов соответственно, $E$ - напряженность продольного электрического поля, $V_{s j}(\varepsilon), E_{s j}(\varepsilon)$ - статические зависимости дрейфовой скорости электронов и напряженности продольного электрического поля от энергии $\varepsilon=\varepsilon(E)$. Для каждого слоя структуры с индексом $j$ эти зависимости можно получить по методике [9] из результатов расчетов стационарных полевых зависимостей дрейфовой скорости $V_{s j}(\varepsilon)$ и энергии $\varepsilon_{s j}(E)$ для объемных материалов методом Монте-Карло [10]. $V_{s}(E)$ - статическая величина дрейфовой скорости электронов в структуре.

Эффективная масса электронов и частота их рассеяния зависят от вероятности их нахождения в каждом слое, которая в свою очередь строго определяется из решения самосогласованных уравнений Шредингера и Пуассона и зависит от температуры электронов. Температура горячих электронов определяется в приближении квазимаксвелловской функции распределения по их энергии, которая представляется как сумма двух слагаемых (условно „тепловой энергии“ и „кинетической энергии направленного движения“"):

$$
\varepsilon=\frac{3}{2} k_{\mathrm{B}} T_{e}+\frac{m^{*} V^{2}}{2}
$$

Температура горячих электронов позволяет определить их долю, перешедшую в $L$-долину. Для этих электронов и электронов в Г-долине в направлении, перпендикулярном поверхности гетероструктуры, можно записать и решить систему самосогласованных уравнений Шредингера и Пуассона для заданного напряжения на затворе, а после этого пересчитать вероятности нахождения электронов по слоям в узкозонном и широкозонном материалах. Однако простые оценки можно провести без усложнения модели. Для этого сделаем следующие предположения.

1. Все электроны, перешедшие в верхние долины, движутся только по узкозонному материалу. Как отмечалось ранее, это в существенно большей мере справедливо для структур с донорно-акцепторным легированием.

2. Пространственное перераспределение электронов из-за переходов в верхнюю долину слабо сказывается на потенциальном рельефе канала. Это заведомо справедливо в достаточно слабых полях, когда бо́льшая часть электронов движется по Г-долине.

Пусть при температуре $T$ в однородном поле вероятность нахождения электронов в $L$-долине для узкозонного материала равна $p_{L}(T)$. Пусть также для этой температуры доля электронов в расчетах только с учетом Г-долины в узкозонном материале $p_{01}(T)$, а в широкозонном $-p_{02}(T)$. Предположим, что все электроны, перешедшие в $L$-долину, движутся только по узкозонному материалу. Вероятность нахождения электронов $p_{2}$ в широкозонном материале будет равна

$$
p_{2}=p_{02}(T)\left(1-p_{L}(T)\right),
$$

а в узкозонном с учетом того, что $p_{01}(T)=1-p_{02}(T)$,

$$
\begin{aligned}
p_{1} & =1-p_{2}=1-p_{02}(T)\left(1-p_{L}(T)\right) \\
& =p_{01}(T)+p_{02}(T) p_{L}(T)
\end{aligned}
$$

Именно эти вероятности и подставляются в уравнения (1)-(4). Данная модель легко обобщается на произвольное число разных слоев в гетероструктуре.

C увеличением доли электронов в узкозонном материале суммарная интенсивность их рассеяния падает, что должно приводить к увеличению дрейфовой скорости электронов. Должен меняться и всплеск
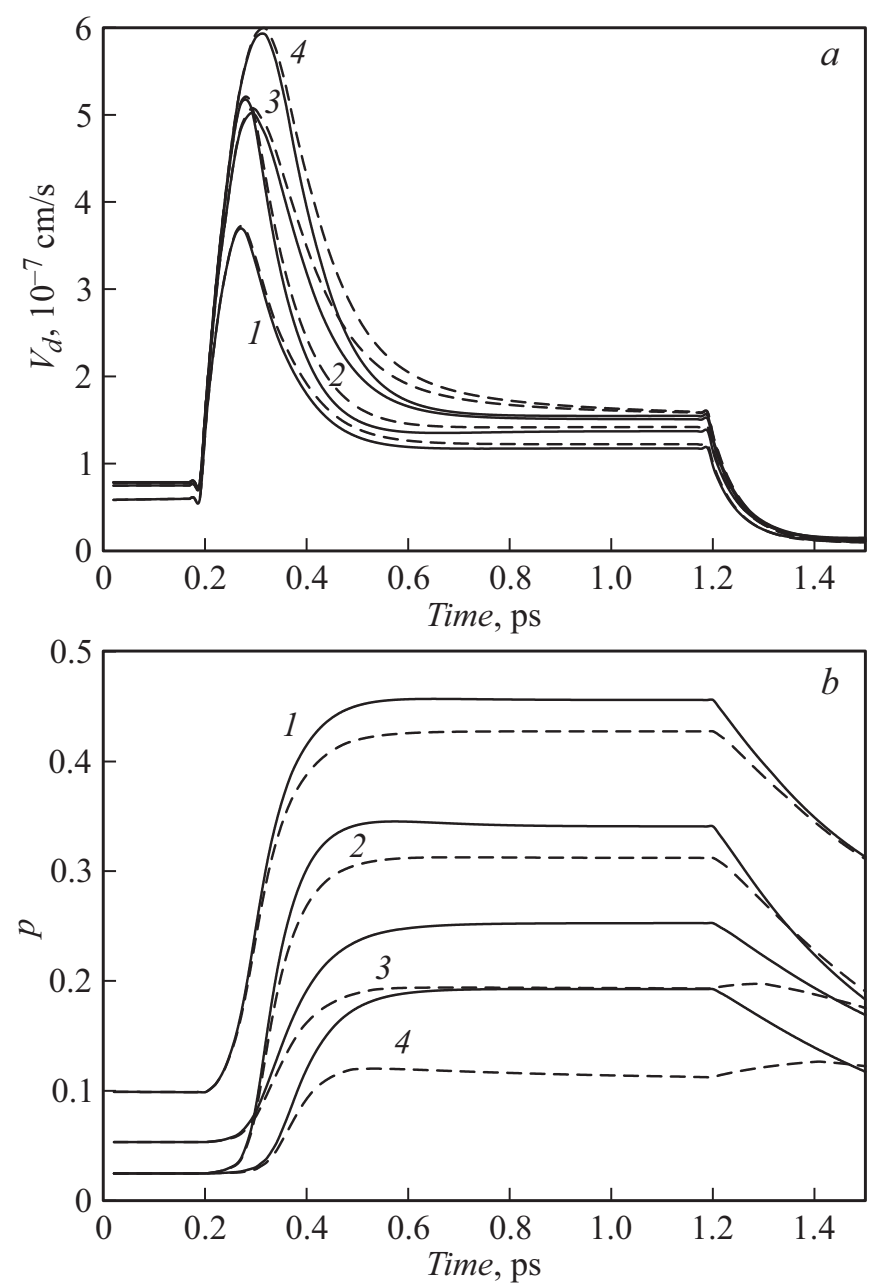

Рис. 1. Зависимости дрейфовой скорости электронов $(a)$ и вероятности нахождения электронов в широкозонном материале $(b)$ от времени. 1 - DpHEMT, $2-$ DmHEMT, $3-$ DA-DpHEMT, 4 - DA-DmHEMT. Штриховые линии - расчет с учетом переходов в $L$-долину, сплошные - расчет без их учета. 


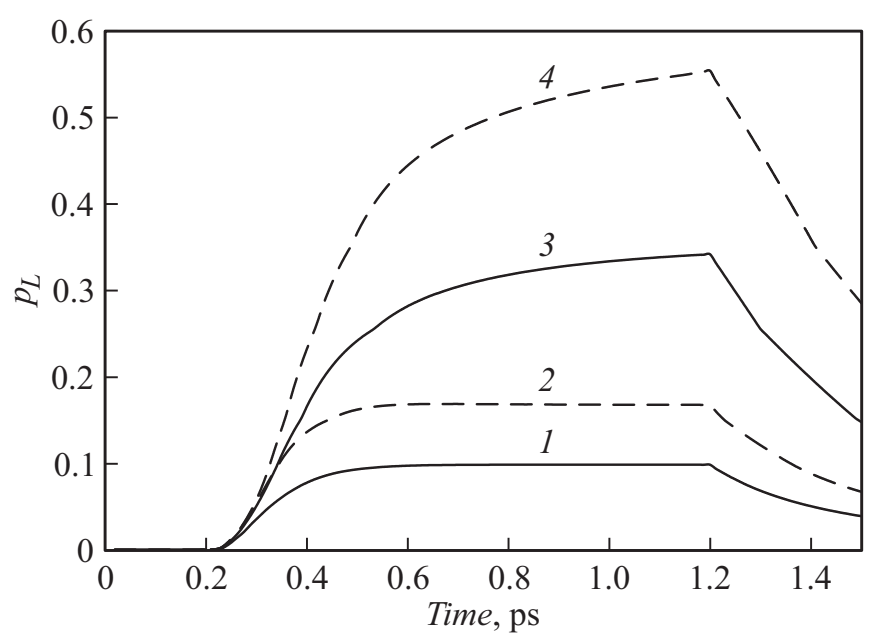

Рис. 2. Зависимость вероятности нахождения электронов в $L$-долине от времени. 1 - DpHEMT, 2 - DmHEMT, 3 DA-DpHEMT, 4 - DA-DmHEMT.

дрейфовой скорости, который сильно зависит от вероятности нахождения в широкозонном материале. На первый взгляд эффект должен проявляться тем сильнее, чем больше электронов переходит в широкозонный материал, т.е. в обычных DpHEMT-структурах. Однако непосредственный расчет демонстрирует прямо противоположное.

На рис. 1 приведены зависимости дрейфовой скорости электронов и вероятности их нахождения в широкозонном материале от времени при их влете в область сильного поля для четырех различных видов транзисторных гетероструктур с двусторонним легированием канала. Зависимости представлены как с учетом усиления локализации электронов верхних долин в канале, так и без него. Расчет проведен для движения электронов в поле с напряженностью $1 \mathrm{kV} / \mathrm{cm}$ в течение $0.2 \mathrm{ps}$ с последующей подачей прямоугольного импульса с амплитудой $30 \mathrm{kV} / \mathrm{cm}$ и длительностью $1 \mathrm{ps,} \mathrm{после}$ чего напряженность поля падает до $1 \mathrm{kV} / \mathrm{cm}$. Расстояние от затвора до канала $3500 \AA$, толщины спейсеров $20 \AA$, ширина квантовой ямы канала $170 \AA$. Классические DpHEMT-структуры предполагались $\delta$-легированными с поверхностной концентрацией доноров $n_{s}=2 \cdot 10^{12} \mathrm{~cm}^{-2}$ в каждом $\delta$-слое. Для симметричных структур DA-DpHEMT с поверхностной плотностью акцепторов $p_{s}=6 \cdot 10^{12} \mathrm{~cm}^{-2}$ поверхностная плотность доноров в $\delta$-слоях задавалась одинаковой и равной $n_{s}=8 \cdot 10^{12} \mathrm{~cm}^{-2}$ в каждом $\delta$-слое. Расстояние между $\delta$ слоем доноров и соседним слоем акцепторов составляло 50 А̊. Структуры моделировались тремя слоями: слой узкозонного канала - нелегированный GaAs, слой сильно легированного $\mathrm{Al}_{0.3} \mathrm{Ga}_{0.7} \mathrm{As}$ и нелегированный спейсер.

Также рассматривались структуры металл$\mathrm{In}_{0.2} \mathrm{Al}_{0.8} \mathrm{As}-\mathrm{In}_{0.2} \mathrm{Ga}_{0.8} \mathrm{As}-\mathrm{In}_{0.2} \mathrm{Al}_{0.8} \mathrm{As}$ (DmHEMT) c более высокими потенциальными барьерами на границе гетероперехода $(\Delta=0.5 \mathrm{eV})[11]$ как в обычном исполнении (с теми же параметрами слоев, что и DpHEMT), так и в донорно-акцепторном исполнении (DA-DmHEMT).

В обычных структурах вероятность нахождения электронов в широкозонном материале в сильном поле заметно выше, чем в структурах с донорно-акцепторным легированием. Несмотря на это, даже полная локализация электронов верхних долин в канале из узкозонного полупроводника слабо сказывается в расчетах на их дрейфовой скорости. Как отмечалось выше, на практике для обычных структур этот эффект будет еще меньше. B то же время в структуре DA-DpHEMT изменение дрейфовой скорости за счет эффекта локализации электронов верхних долин в узкозонном канале оказывается заметным, еще выше оно в DA-DmHEMT. Там ее рост может достигать более чем $15 \%$. И хотя на фоне увеличения от введения донорно-акцепторного легирования как статической дрейфовой скорости электронов, так и ее всплеска [7,12] этот эффект и невелик, он может оказать определенное влияние на рост тока в DA-DpHEMT и DA-DmHEMT. Разницу можно объяснить следующим образом. В обычных DpHEMT-структурах горячие электроны достаточно интенсивно переходят в широкозонный материал (рис. $1, b$ ) и там теряют энергию и скорость, вследствие этого их доля в $L$-долине (рис. 2) оказывается меньше. Соответственно меньше электронов локализуется в канале (рис. 1,b). Рассеяние в широкозонном материале намного интенсивнее, чем в узкозонном. В связи с этим для оценки эффекта важна не абсолютная разница в величине вероятности нахождения электронов в широкозонном материале в расчетах с учетом и без учета конденсации, а относительная. По данному критерию эти вероятности в DpHEMT-структурах (кривые 1 на рис. $1, b$ ) близки; соответственно мало влияние конденсации на дрейф электронов. В структурах с донорно-акцепторным легированием все наоборот. Доля электронов в широкозонном материале гораздо меньше - выше их температура, больше доля в $L$-долине, больше электронов конденсируется в канале. Относительное изменение числа электронов в широкозонном материале гораздо больше (кривые 3,4 на рис. $1, b$ ). Соответственно больше и влияние локализации электронов на величину дрейфовой скорости.

Таким образом, на основе теоретических оценок показано, что локализация горячих электронов, перешедших в верхние долины, в узкозонном канале транзистора может быть дополнительным механизмом увеличения тока в структурах с донорно-акцепторным легированием. В зависимости от параметров гетероструктуры учет этого эффекта при моделировании может приводить к увеличению величины дрейфовой скорости до 15\% по сравнению с расчетами без его учета.

\section{Конфликт интересов}

Авторы заявляют, что у них нет конфликта интересов. 


\section{Список литературы}

[1] Glisson T.H., Hauser J.R., Littlejohn M. // J. Appl. Phys. 1980. V. 51. N 10. P. 5445-5449.

[2] Sakamoto R., Akai K., Inoue M. // IEEE Trans. Electron Dev. 1989. V. 36. N 10. P. 2344-2352.

[3] Кальба А.А., Пашковский А.Б. // ФТП. 1990. Т. 24. В. 3. C. $521-526$.

[4] Вагидов Н.3., Грибников З.С., Иващенко В.М. // ФТП. 1989. T. 23. B. 2. C. 304-311.

[5] Вагидов Н.3., Грибников З.С., Иващенко В.М. // ФТП. 1990. T. 24. B. 6. C. 1087-1094.

[6] Горфинкель В.Б., Шофман С.Г. // ФТП. 1988. Т. 22. В. 5. C. $793-798$.

[7] Пашковский А.Б., Новиков С.И., Лапин В.Г., Лукашин В.М., Мартынов Я.Б. // Письма в ЖТФ. 2018. Т. 44. B. 17. C. 103-110.

[8] Лукашин В.М., Пашковский А.Б., Журавлев К.С., Торопов А.И., Лапин В.Г., Соколов А.Б. // Письма в ЖТФ. 2012. T. 38. B. 17. C. $84-89$.

[9] Shur M. // Electron. Lett. 1976. V. 12. N 23. P. 615-616.

[10] Гарматин А.В. // Электронная техника. Сер. 1. Электроника СВЧ. 1985. № 3(377). С. 66-68.

[11] Василевский И.С., Виниченко А.Н., Каргин Н.И. // 8-я Междунар. науч.-практ. конф. по физике и технологии наногетероструктурной СВЧ-электроники. Мокеровские чтения. Тез. докл. М., 2017. С. 28-29.

[12] Протасов Д.Ю., Гуляев Д.В., Бакаров А.К., Торопов А.И., Ерофеев Е.В., Журавлев К.С. // Письма в ЖТФ. 2018. T. 44. B. 6. C. $77-84$. 